\title{
A NOTE ON THE SIGNIFICANCE OF THE VEGETATIVE NERVOUS SYSTEM IN TABES DORSALIS.
}

\author{
BY MARY R. BARKAS, LONDON.
}

Even in a disease as common and as exhaustively studied as tabes dorsalis, we are still far from being able to explain in a completely satisfactory manner all of its manifestations; it seems worth while, therefore, to consider its problems from a point of view which much recent research has brought into prominence, namely, that of the influence of the vegetative nervous system upon the localization and symptomatology of nervous diseases.

Orr and Rows ${ }^{1}$ have shown how great is the part played by the autonomic nerve supply in determining the localization of lesions caused by infective or toxic agents acting on the central nervous system ; a deficiency of adrenalin supply (in Addison's disease) or a metabolic disorder (such as pernicious anæmia) may be associated with lesions in the spinal cord which can best be explained as being determined by a dysfunction of the sympathetic nerve supply to the pial vessels, which, in a pathologically dilated condition, allow free access for some toxic substance to the neurones of the spinal cord. In view of their findings, it seems very possible that the same factor may be at work in tabes determining the site of election for the lesions of this disease.

Can any support be found for this theory in the clinical picture of the affection? Certain of its symptoms point indisputably to an involvement of the autonomic nervous system and cannot be explained by the well-known lesions of the somatic nervous system-the visceral crises, the dysfunction of sphincters and genitalia at a time when their sensation is not lost, the pallid complexion, the ptosis, the absent ciliospinal reflex, and the contracted pupil which still reacts to light, so often found in the early stages of the disease. Other symptoms and manifestations are accepted as due to some ill-defined 'trophic changes' or to sensory loss, but these so-called explanations are far from satisfactory. The Charcot joint, for example, is probably not wholly due to a lack of pain sensation and consequent injury, though this may account for the complete disintegration of the later stages; but in the beginning such joints are often very painful, and neither loss of muscle tone nor analgesia can be wholly responsible for the condition. Trophic 
changes, abnormalities in the supply of nourishment, and the proper maintenance of tissue metabolism, are essentially the province of the autonomic system, which certainly controls the blood supply at least; hence, if these be the cause of the disorder, we must consider the autonomic nervous system to be a predominant factor. The same argument applies to the perforating ulcer.

The sensory disturbances of the disease are generally regarded as the result of the lesions found in the posterior columns of the spinal cord and the posterior root ganglia, and to a great extent this is certainly true; but these lesions affect not only the somatic but also the autonomic afferent neurones, both of which have their trophic centres in the posterior root ganglia. It was shown as long ago as 1898 by the late Dr. F. E. Batten, that the muscle spindles and their nerve supply are degenerated in tabes, and the ataxy and loss of muscle sensibility were correlated by him with these findings. But it has more recently been shown by the late Professor John Hunter ${ }^{2}$ and others, that the muscle spindle is supplied by autonomic as well as by somatic nerves, and in view of the pain produced in visceral crises by irritation of the autonomic afferent nerves, it is only reasonable to suppose that these are also responsible in part for the lightning pains and loss of deep pain in the muscles, as well as for some, at least, of the loss of muscle tone.

The distribution of the hyperalgesia and analgesia in tabes affords some support for this view. It has never been satisfactorily explained why just those segmental areas are first and most commonly affected which correspond to the outflow from the cord of the sympathetic neurones, C8 to L2, and of these, pre-eminently the upper dorsal segments. Orr and Rows ${ }^{1}$ remark that this region is first and most severely affected in Addison's disease and in subacute combined degeneration; they cannot explain why this should be the case, and believe that only further researches into the phylogeny and ontogeny of the whole nervous system and ductless glands will elucidate the matter. The clinical fact remains, and supports the view that some part, at least, of the sensory disorder in the skin and muscles is due to lesions of the autonomic rather than of the somatic neurones.

Recent researches by Boecke, Langelaan, Hunter ${ }^{2}$ and others, have proved indisputably that not only the viscera and involuntary musculature, but also the striated muscles, receive a nerve supply from the autonomic system. The striated muscles contain two types of fibre, the coarsely striated thicker fibres having a somatic nerve supply, and subserving the function of quick contraction, while the thinner, more irregularly striated fibres receive a sympathetic efferent nerve and are mainly concerned with the preservation of posture and the maintenance of plastic tone. Hunter has shown that after section of the sympathetic efferent fibres to a limb there is a loss of postural tone and 
an increased freedom of passive movement which strikingly resembles those found in tabes, and these disorders probably play as important a part as the sensory loss in the production of tabetic ataxy. The preservation of the power of quick and forcible voluntary contraction in muscles apparently wasted and toneless agrees with the view of Hunter that the striated muscles contain two systems of fibres differing both in function and in nerve supply. It would be interesting to know whether pathological findings in tabetic muscles show degeneration of the finer, less striated fibres and their nerve supply, as well as of the muscle spindles.

Orr and Rows ${ }^{1}$ plead for a wider recognition of the fact that the somatic and autonomic nervous systems form one indissoluble and interdependent whole, functioning in unison. This note aims at drawing attention to the importance of that one of the partners whose rôle has been somewhat overlooked in a disease which may throw as valuable light on the function of the autonomic nervous system as it has already done on that of the somatic system, and this light may show a path to more effective treatment of tabes dorsalis in its early stages than science has yet provided.

\section{REFERENCES.}

1 ORR and Rows. "The interdependence of the sympathetic and central nervous systems." Brain, 1918, xli.

2 JoH HuNTER (Lectures given by Elliott Smith from unpublished papers). Brit. Med. Jour., 1925, Jan. 31, et seq. 